\section{ENGINEERED TOXIN BODY MEDIATED DEPLETION OF TIGIT EXPRESSING IMMUNE CELLS FOR CANCER IMMUNOTHERAPY}

Elizabeth Saputra, Garrett Cornelison, Jennifer Mitchell, Karia Williams, Andrea Mendiola, Rachael Orlandella, John Majercak, Joseph Dekker, Chris Moore, Swati Khanna*. Molecular Templates Inc., Austin, TX, USA

Background TIGIT (T cell immunoreceptor with Ig and ITIM domains) is an exciting novel target for immuno-oncology which functions as an immune checkpoint on multiple immune cell types including memory CD8+, CD4+ Treg, and memory CD4+ cells. TIGIT upregulation on tumor infiltrating lymphocytes (TILs) has been observed in multiple cancer types and contributes to an immunosuppressive tumor microenvironment (TME). Interestingly, TIGIT is commonly co-expressed with PD-1 on Tregs in the TME, tumor antigen specific CD8 $+\mathrm{T}$ cells and CD8+ TILs, leading to weakened anti-tumor immune responses. ${ }^{1-2}$ To date, TIGIT inhibiting monoclonal antibodies $(\mathrm{mAb})$ have shown little activity as a monotherapy in clinical and preclinical studies. ${ }^{3-4}$ Therefore, current clinical trials are now focused on combining TIGIT mAbs with known commercial PD-1 or PD-L1 mAbs. A TIGIT-specific engineered toxin body (ETB) represents a wholly new approach to targeting TIGIT expressing cells including those co-expressing TIGIT and PD-1.

Methods ETBs targeting TIGIT were designed to deplete TIGIT-expressing TILs, including Tregs, directly in the TME. ETBs are proteins that consist of an antibody fragment genetically fused to a proprietary de-immunized (DI) form of the Shiga-like toxin A subunit (SLTA). These proteins are specific for a cell surface receptor, and function through triggering rapid internalization upon binding, followed by an enzymatic and irreversible termination of ribosomal protein synthesis resulting in cellular apoptosis. Here we provide proof of concept for ETBs as a novel modality for the depletion of TIGIT-expressing immune cells.

Results TIGIT-targeting ETBs exhibit potent in vitro cytotoxicity of TIGIT over-expressing cell lines $(\mathrm{IC} 50<1 \mathrm{nM})$. These ETBs also lead to apoptotic depletion of ex vivo TIGITexpressing regulatory $\mathrm{T}$ cells (Tregs) from healthy donors. In mixed culture assays, TIGIT ETBs increase the proliferation of TIGIT negative $\mathrm{T}$ cells by depleting TIGIT-expressing $\mathrm{T}$ cells.

Conclusions Studies to assess pharmacodynamics and efficacy of TIGIT targeting ETBs using a double knock-in (TIGIT and PD-1) mouse tumor model are ongoing, but these early proof of concept in vitro data support the hypothesis that ETBs can deplete TIGIT positive immune cell populations including those co-expressing PD-1. It is possible that targeted TIGIT inhibition through ETB-induced cell death could tip the balance towards tumor regression by eliminating this novel checkpoint (and TIGIT/PD-1 co-expression) at the level of the TME.

\section{REFERENCES}

1. Jinhua $X$, Ji W, Shouliang $C$, Liangfeng $Z$. Expression of immune checkpoints in $T$ cells of esophageal cancer patients. Oncotarget 2016;7(39):1-10.

2. Blessin NC, Simon R, Kluth M, Fischer $K$, et al. Patterns of TIGIT expression in lymphatic tissue, inflammation and cancer. Dis Markers 2019:2019:1-13.

3. Johnston RJ, Comps-Agrar L, Hackney J, Yu X, et al. The immunoreceptor TIGIT regulates anti-tumor and antiviral CD8(+) T effector function. Cancer Cell 2014;26(6):923-927.

4. Bendell JC, Bedrad P, Bang Y-J, LoRusso $P$, et al. Phase la/lb dose-escalation study of the anti-TIGIT antibody Tiragolumab as a single agent and in combination with atezolizumab in patients with advanced solid tumors. Proceedings: AACR Annual Meeting 2020; April 27-28, 2020 and June 22-24, 2020; Philadelphia, PA. 